\title{
Analyzing Software Engineering Experiments: Everything You Always Wanted to Know but Were Afraid to Ask
}

\author{
Natalia Juristo $^{1,2}$, Sira Vegas ${ }^{1}$ \\ ${ }^{1}$ Escuela Técnica Superior de Ingenieros Informáticos \\ Universidad Politécnica de Madrid, Spain \\ \{natalia,svegas\}@fi.upm.es \\ ${ }^{2} \mathrm{M}$-Group \\ Department of Information Processing Science \\ University of Oulu, Finland
}

\begin{abstract}
Experimentation is a key issue in science and engineering. But it is one of software engineering's stumbling blocks. Quite a lot of experiments are run nowadays, but it is a risky business. Software engineering has some special features, leading to some experimentation issues being conceived of differently than in other disciplines. The aim of this technical briefing is to help participants to avoid common pitfalls when analyzing the results of software engineering experiments. The technical briefing is not intended as a data analysis course, because there is already plenty of literature on this subject. It reviews several issues that we have identified in published SE experiments.
\end{abstract}

\section{Categories and Subject Descriptors \\ D.2.0 [Software Engineering]: General}

\section{General Terms}

Experimentation.

\section{Keywords}

Software Engineering experimentation, controlled experiments, analysis of experiments.

\section{DESCRIPTION OF THE TOPIC}

The goal of this technical briefing is to help participants to improve how they analyze the data when running controlled experiments.

The technical briefing starts by recalling what an experiment is, and its distinctive features: control and causality. This introduction will last 5 minutes (Natalia).

It then explores, by means of a real example, different design alternatives and their impact in data analysis. This will last 10 minutes (Natalia).

Then, four topics related to data analysis will be explored. Advice

Permission to make digital or hard copies of all or part of this work for personal or classroom use is granted without fee provided that copies are not made or distributed for profit or commercial advantage and that copies bear this notice and the full citation on the first page. To copy otherwise, or republish, to post on servers or to redistribute to lists, requires prior specific permission and/or a fee.

ICSE'16 Companion, May 14-22, 2016, Austin, TX, USA.

ACM xxx-xxx-x-xxxxx.

http://dx.doi.org/xx.xxxx/xxxxxxxxxx will be given to participants based on our own experiences after more than 15 years running controlled experiments [1]. The real experiment presented in the introduction will be used during the whole technical briefing, to illustrate the issues covered:

1. One-tailed vs. two-tailed tests. SE experimenters very often opt for a one-tailed hypothesis, but this can be a shortcoming in many experiments. We will discuss when each type should be used (Natalia, 10 minutes).

2. Choosing the right statistical model. The statistical model to be chosen should match the experimental design. We will discuss two designs which are commonly not properly analyzed: blocked and crossover designs (Sira, 15 minutes).

3. Parametric vs. non-parametric tests. We will discuss the different options that can be used when data do not meet the parametric tests assumptions: transformations and nonparametric tests (Sira, 20 minutes).

4. The $\mathbf{3}$ musketeers: statistical significance, effect size and power. We will discuss the meaning and implications of each one, how the three parameters relate to each other, and how they should be used to properly interpret the results of an experiment. Non-significant results might be due to lack of power. Statistical significant results might be not relevant due to small effect sizes, etc. (Sira, 20 minutes).

The technical briefing will end with participants' questions (Natalia, Sira, 10 minutes).

\section{INTEREST OF THE TOPIC FOR THE SE COMMUNITY}

It is now very common practice to conduct laboratory experiments in SE. However, this is a challenging error-prone activity. Shepperd, Bowes and Hall [3] analyzed the results of 42 papers reporting studies comparing methods for predicting faultproneness. They found that the explanatory factor that accounted for the largest percentage of the differences between studies $(30 \%)$ was research group [3]. In contrast, the main topic of research, accounted for only $1.3 \%$ of the variation between the studies. Sjøberg et al. [4] reported that replications performed by researchers who undertook the initial study were more likely to find the same result than replications undertaken by independent researchers. In the field of psychology, Pashler and Wagenmakers [2] report "a crisis of confidence in psychological science reflecting an unprecedented level of doubt among practitioners about the reliability of research findings in the field".

Experimentation is quite a recent practice in SE (compared with 
other much more mature experimental disciplines). We still have a long way to go, and much more effort and research is needed to adapt the experimental paradigm to SE. Experimentalism is a paradigm that needs to be instantiated, translated and adapted to the idiosyncrasy of each experimental discipline. Copy and paste, that is, copy from physics what an experiment is, copy from medicine the threats to the validity of experiments, or copy from psychology how to deal with experimental subjects, will not do. We can borrow from other experimental disciplines, but our field needs to adopt its own form of experimentalism.

The technical briefing focuses on aspects of SE experiment analysis that often are error prone.

\section{PRESENTERS' BACKGROUND}

\subsection{Bios}

Natalia Juristo is full professor of software engineering at the Universidad Politécnica de Madrid (Spain) and FiDiPro (Finland Distinguished Professor Program) professor at the University of Oulu (Finland). Her research interests are experimental software engineering, requirements and testing.

Sira Vegas is associate professor of software engineering at the Universidad Politécnica de Madrid (Spain). Her research interests are experimental software engineering and software testing.

\subsection{Empirical SE Tutorials Taught}

- N. Juristo, S. Vegas. Challenges of Conducting Software Engineering Experiments: Everything You Always Wanted to Know but Were Afraid to Ask. Half day. $10^{\text {th }}$ joint meeting of the European Software Engineering Conference and the Symposium on the Foundations on Software Engineering (ESEC/FSE 2015).

- O. Dieste, A. Jedlitschka, N. Juristo, D. Mendez. In Vivo Experimentation in Software Engineering. Full day. $12^{\text {th }}$ International Advanced School on Empirical Software Engineering (IASESE 2014).

- O. Dieste, N. Juristo, S. Vegas. Replication and Aggregation of Software Engineering Experiments. Full day. $6^{\text {th }}$ International Advanced School on Empirical Software Engineering (IASESE 2008).

- A. Moreno, S. Vegas. Experimentation in Software Engineering. Half day. II Jornadas Iberoamericanas de Ingeniería del Software e Ingeniería del Conocimiento (JISIC 2002).

\subsection{Empirical SE Keynotes and Invited Talks}

- S. Vegas. What Makes a Good Empirical Software Engineering Thesis?: Some Advice. $13^{\text {th }}$ International Doctoral Symposium on Empirical Software Engineering (IDoESE 2015).

- N. Juristo. Basics on Design and Analysis of SE Experiments:
Widespread Shortcomings. Doctoral Symposium at the $36^{\text {th }}$ International Conference on Software Engineering (DS-ICSE 2014).

- N. Juristo. Towards Understanding Replication of Software Engineering Experiments. $7^{\text {th }}$ International Symposium on Empirical Software Engineering and Measurement (ESEM 2013).

- N. Juristo. The Importance Experiments and Empirical Aspects in Computer Science. $8^{\text {th }}$ European Computer Science Summit (ECSS 2012).

- S. Vegas. Using Differences among Replications of Software Engineering Experiments to Gain Knowledge. 7th Working conference on Mining Software Repositories (MSR 2010).

- N. Juristo. How Science can Support Software Engineering. Evaluation and Assesment on Software Engineering Conference (EASE 2010.)

- N. Juristo. Gaining Evidences in SE: Enabling Collaborative Empirical Research. $1^{\text {st }}$ Experimental Software Engineering Latin American Workshop (ESELAW), 2004.

\section{TECHNICAL BRIEFING HISTORY}

The contents of this technical briefing are based on the one-hour talk given by Natalia Juristo at the ICSE 2014 Doctoral Symposium, entitled "Basics on Design and Analysis of SE Experiments: Widespread Shortcomings", and covers some of the topics (the ones related to the analysis of experimental data) taught on the more general four-hour tutorial given by Natalia Juristo and Sira Vegas at the ESEC/FSE 2015, entitled "Challenges of Conducting Software Engineering Experiments: Everything You Always Wanted to Know but Were Afraid to Ask" (13 attendees).

\section{ACKNOWLEDGMENTS}

Research funded by the Spanish Ministry of Economy and Competitiveness research grant TIN2014-60490-P.

\section{REFERENCES}

[1] N. Juristo, A.M. Moreno. Basics of Software Engineering Experimentation. Kluwer, 2001.

[2] H. Pashler, E.J. Wagenmakers. Editors' Introduction to the Special Section on Replicability in Psychological Science: A Crisis of Confidence? Perspectives on Psychological Science, 7(6):528$530,2012$.

[3] M. Shepperd, D. Bowes, T. Hall. Researcher Bias: The Use of Machine Learning in Software Defect Prediction. IEEE Transactions on Software Engineering, 40(6):603-616, 2014.

[4] D.I.K. Sjøberg, J.E. Hannay, O. Hansen, V.B. Kampenes, A. Karahasanovic, N.K. Liborg, A.C. Rekdal. A survey of controlled experiments in software engineering. IEEE Transactions on Software Engineering, 31(9):733-753, 2005. 